\title{
STEERABLE PYRAMID FILTERS FOR SELECTIVE IMAGE ENHANCEMENT APPLICATIONS
}

\author{
Q. Wu, M. A. Schulze, K. R. Castleman \\ Perceptive Scientific Instruments, Inc. \\ 2525 South Shore Blvd. Ste. 100 \\ League City, TX 77573, USA
}

\begin{abstract}
In this paper we describe a method we developed for selective detection and enhancement of image feature patterns. A set of directional basis filters were designed to detect the image features of interest, and a steerable pyramid transform [1,2] is used to generate a multiscale representation of the image features that has desirable shift and rotation invariance. By analyzing and selectively processing the transform coefficients, image feature detection and enhancement can be achieved with designed flexibility in scale, orientation, location, and degree of enhancement. Unlike traditional techniques, this new method promises the selectivity and flexibility desirable in many image enhancement applications. Preliminary experimental results are presented to exemplify the feasibility of the approach.
\end{abstract}

\section{Introduction}

Traditional image enhancement techniques employing conventional convolution filtering and contrast stretching lack selectivity, and flexibility. Such selectivity and flexibility are desired in many image analysis applications. A example in biomedical image analysis would be that a cytogeneticist desires to enhance chromosome bands at a particular scale and in certain orientation and position for designated DNA sequence analysis. This is not achievable by the traditional image enhancement techniques because of the lack of spatial and scale selectivity of the conventional convolution filtering and contrast stretching operations.

The inability of traditional techniques for selective detection and enhancement of image features can be intuitively explained by the fact that the design of conventional convolution filters, in general, is based on the representation of images in the frequency domain. A bandpass filtering, for instance, corresponds to masking out the portion of the passband image spectrum. Thus it is not difficult to see the intrinsic limitation of the approach, as this offers no space-frequency localization of image features. To detect and enhance image features at a different scale, the convolution filter would have to be redesigned and the designer would have to try different passbands before a new filter kernel can be derived.

Filter bank and pyramid transforms, on the other hand, offer appealing means of multiscale image representation. In addition to this, they also offer means to characterize and localize image features in both space and transform domains. Figure 1 shows the steerable pyramid transform of an image with five human chromosomes. These chromosomes are well characterized and localized at different scale, orientation, and position by the transform coefficients in the representation. Selective detection and enhancement of the chromosome image features at various level of detail, therefore, become possible and straight forward by analyzing and processing the transform coefficient image at designated scale, orientation, and location, provided that the basis filters are properly designed to detect the features.
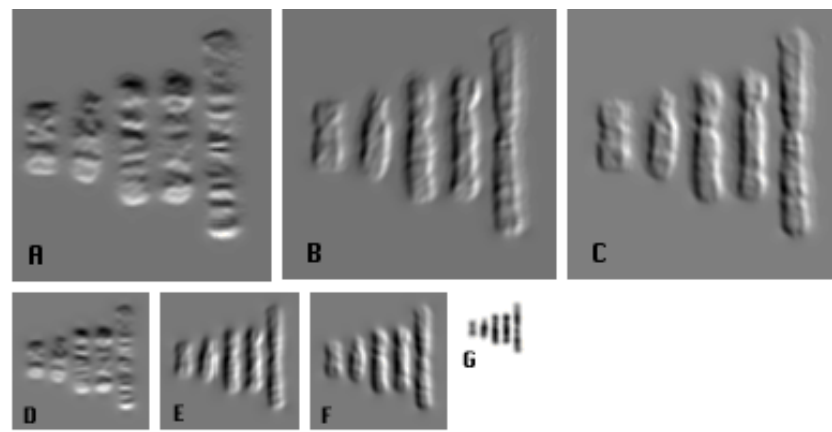

Figure 1. Steerable pyramid transform of the human chromosome image.

Most multiscale image transforms, however, are known to suffer from the shift and rotation variance problem. This affects their applicability to image enhancement. To overcome this problem we adopt the steerable pyramid transform that promises desirable property of shift and rotation invariance [1-5]. In the following, we first introduce the design of the basis filters for detection of image concavity/convexity features, and then describe how we achieve selective enhancement of these image features using a multiscale analysis and processing procedure. Finally we show some preliminary experimental results that exemplify the feasibility of the proposed approach.

\section{Design of Steerable Pyramid Filters}

The design of basis filters is a crucial step in constructing a steerable pyramid transform. The idea is to design the filters so that the image features of interest can be saliently characterized in the multiscale representation of the image. Gradient filters, for instance, can be used to localize boundaries of objects in an image. Second derivative filters, on the other hand, are good at characterizing image brightness concave/convex regions. Here we introduce the design of the second derivative filters for detecting and enhancing image concavity/convexity.

We developed a method to design the steerable pyramid basis filters conveniently in the frequency domain [6]. Subject to the 
spectral constraints of the steerable filters outlined in [3], these filters are designed in polar coordinates as follows:

1. First lowpass filter:

$$
L_{1}(\omega)=\left\{\begin{array}{cc}
1, & |\omega|<\omega_{1} \\
\sqrt{\frac{1}{2}\left[1+\cos \left(\frac{\omega-\omega_{1}}{\omega_{\max }-\omega_{1}} \cdot \pi\right)\right]}, & \omega_{1} \leq|\omega| \leq \omega_{\max } \\
0, & |\omega|>\omega_{\max }
\end{array}\right\} .
$$

where $\omega_{\max }$ is the bandlimit of the image and $\omega_{1}$ is the frequency $L_{1}(\omega)$ starts to attenuate.

2. Highpass filter:

$$
H(\omega)=\left\{\begin{array}{cc}
0, & |\omega|<\omega_{1} \\
\sqrt{\frac{1}{2}\left[1-\cos \left(\frac{\omega-\omega_{1}}{\omega_{\max }-\omega_{1}} \cdot \pi\right)\right]} & \omega_{1} \leq|\omega| \leq \omega_{\max } \\
1, & |\omega|>\omega_{\max }
\end{array}\right\} .
$$

3. Second lowpass filter:

$$
L_{2}(\omega)=\left\{\begin{array}{cc}
1, & |\omega|<\omega_{0} \\
\sqrt{\frac{1}{2}\left[1+\cos \left(\frac{\omega-\omega_{0}}{\omega_{1}-\omega_{0}} \cdot \pi\right)\right],} & \omega_{0} \leq|\omega| \leq \omega_{1} \\
0, & |\omega|>\omega_{1}
\end{array}\right\} .
$$

where $\omega_{0}$ is the frequency $L_{2}(\omega)$ starts to attenuate.

4. Bandpass filters:

$$
R(\omega)=\left\{\begin{array}{cc}
0, & |\omega|<\omega_{0} \\
\sqrt{\frac{1}{2}\left[1-\cos \left(\frac{\omega-\omega_{0}}{\omega_{1}-\omega_{0}} \cdot \pi\right)\right],} & \omega_{0} \leq|\omega| \leq \omega_{1} \\
0, & \omega_{1}<|\omega|<\omega_{\max }
\end{array}\right\} .
$$

Since the minimum number of orientation bands to form the second derivative steerable pyramid transform is $N_{\text {min }}=3[1,2]$, the magnitude spectra of the $i$ th oriented bandpass filters become

$$
B_{i}(\omega, \theta)=R(\omega) \cos ^{2}\left(\theta-\frac{2 \pi \cdot i}{N}\right), \quad i=0,1,2 .
$$

where $\cos ^{2}\left(\theta-\frac{2 \pi \cdot i}{N}\right)$ is the angular component. The lowpass and highpass filters $L_{1}, H$, and $L_{2}$ are circularly symmetrical about the origin.

Figure 2 shows 3-D surface plots of these filters in the frequency domain. By inverse Fourier transform we can obtain the impulse response of these filters. With proper truncation they constitute the basis filter kernels for space domain convolution.

\section{Image Feature Detection and Enhancement}

Performing the steerable pyramid image transform using the basis filters designed in (1) - (5), a multiscale representation of the image can be generated as in Figure 1, in which bandpass filtered images at different decomposition levels correspond to three oriented second derivative images at that scale. This representation is well suited for selective detection and enhancement of image features that correspond to image brightness concave/convex regions at different scale, location, and orientation.
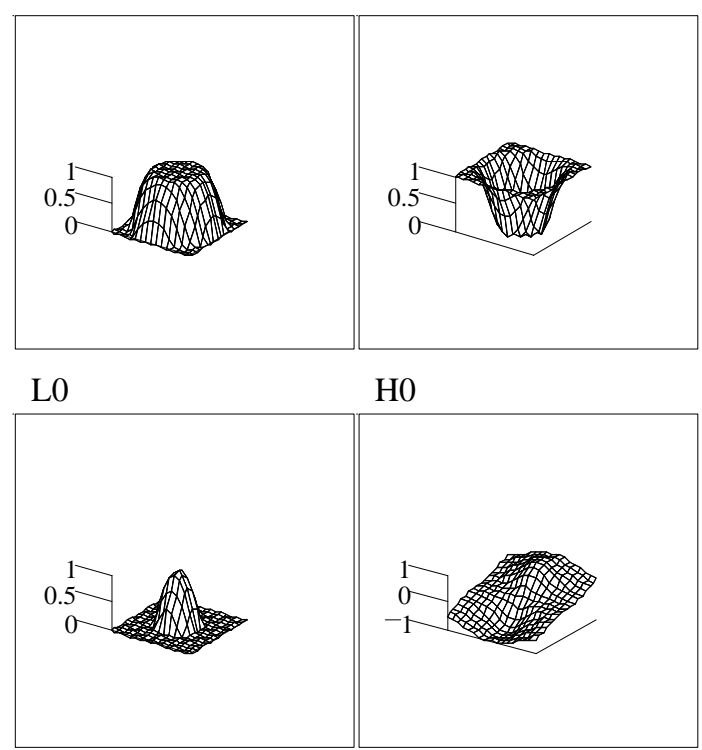

L1

B1

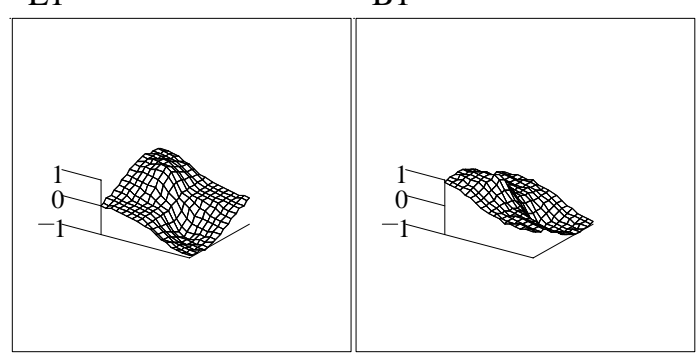

B2

B3

Figure 2. Surface plots of the designed basis filters in the frequency domain.

Since the image concavity/convexity can be located accurately by finding the zero-crossings of the second derivative image, enhancement of these regions can be achieved by stretching the sets of pixels in the second derivative image that correspond to the peaks of the regions, and using these pixels to accentuate the contrast around the regions.

Let us denote the original image as $f(x, y)$, the bandpass filtered image at decomposition level $i$ in orientation $j$ as $\Delta_{i}^{j} f(x, y)$, and the reconstructed image at level $i$ as $f_{i}(x, y)$. The bandpass filtered image at decomposition level $i$ in orientation $\phi$ is

$$
\Delta_{i}^{\phi} f(x, y)=\sum_{j=0}^{N_{\min }-1} k_{j}(\phi) \Delta_{i}^{j} f(x, y) .
$$


where the interpolation functions $k_{j}(\phi)$ is derived by solving the equations given in the steerability theorem $[1,2]$ :

$$
k_{j}(\phi)=\frac{1}{3}\left[1+2 \cos \left(2\left(\phi-\frac{2 \pi \cdot j}{N}\right)\right)\right], \quad j=0, \ldots, N_{\min }-1 .
$$

Suppose a convex region in the image is oriented in $\phi$. It can be detected by finding a negative peak in $\Delta_{i}^{\phi} f(x, y)$, and can be located precisely by finding the two corresponding zerocrossings. The enhancement of the region can be achieved by performing the following procedure:

(a). Clip the image $\Delta_{i}^{\phi} f(x, y)$ to zero out all positive-valued pixels, i.e.,

$$
\begin{aligned}
& \left\{\left[\left(x_{l}, y_{l}\right), \Delta_{i}^{\phi} f\left(x_{l}, y_{l}\right)\right] \mid \Delta_{i}^{\phi} f\left(x_{l}, y_{l}\right)>0\right\} \\
& \rightarrow\left\{\left[\left(x_{l}, y_{l}\right), \Delta_{i}^{\phi} f\left(x_{l}, y_{l}\right)=0\right] \mid \Delta_{i}^{\phi} f\left(x_{l}, y_{l}\right)>0\right\} .
\end{aligned}
$$

(b). Perform linear stretching on the negative-valued pixels in the images, i.e.,

$$
\begin{aligned}
& \left\{\left[\left(x_{l}, y_{l}\right), \Delta_{i}^{\phi} f\left(x_{l}, y_{l}\right)\right] \mid \Delta_{i}^{\phi} f\left(x_{l}, y_{l}\right)<0\right\} \\
& \rightarrow\left\{\left[\left(x_{l}, y_{l}\right), \eta_{i} \cdot \Delta_{i}^{\phi} f\left(x_{l}, y_{l}\right)\right] \mid \Delta_{i}^{\phi} f\left(x_{l}, y_{l}\right)<0\right\} .
\end{aligned}
$$

where $\eta_{i} \geq 0, \eta_{i}$ being the degree of enhancement factor that can vary with scale $i$.

(c). Subtract $\Delta_{i}^{\phi} f\left(x_{l}, y_{l}\right)$ from $f_{i}(x, y)$ to yield $f_{i}{ }^{\prime}(x, y)$, i.e.,

$$
f_{i}{ }^{\prime}(x, y)=f_{i}(x, y)-\Delta_{i}^{\phi} f\left(x_{l}, y_{l}\right) .
$$

(d). Using $f_{i}{ }^{\prime}(x, y)$ instead of $f_{i}(x, y)$ during the reconstruction.

This approach of image concavity/convexity detection and enhancement can be graphically described by the onedimensional illustration shown in Figure 3. Here a convex region is modeled by a Gaussian curve

$$
s(x)=\frac{1}{\sqrt{2 \pi}} e^{-\frac{x^{2}}{2}} .
$$

Its first and second derivative are respectively

$$
s 1(x)=\frac{d}{d x} s(x)=-\frac{1}{\sqrt{2 \pi}} e^{-\frac{x^{2}}{2}}
$$

and

$$
s 2(x)=\frac{d^{2}}{d x^{2}} s(x)=\frac{\left(x^{2}-1\right)}{\sqrt{2 \pi}} e^{-\frac{x^{2}}{2}} .
$$

The enhanced result, denoted by $y(x)$, is computed by:

$$
y(x)=\left\{\begin{array}{cc}
s(x)-\eta \cdot s 2(x), & s 2(x)<0 \\
s(x), & s 2(x) \geq 0
\end{array}\right\} .
$$

where $\eta$ is the degree of enhancement factor. From their curve plots in Figure 3, the principle of concavity/convexity detection and enhancement using the second derivative should be straight forward to understand.

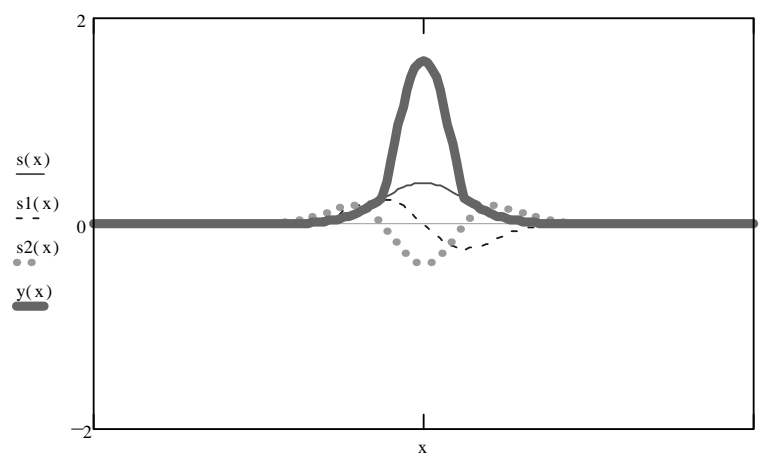

Figure 3. One-dimensional illustration of image convexity detection and enhancement.

\section{Experimental Results}

The proposed method is implemented and tested on a variety of images, in which features of interest are image brightness concave/convex regions. The program performs the steerable pyramid transform on the test images using the basis filters described in section 2 . The user can interactively change the program parameters described in (6) - (10) through a graphical user interface to achieve selective detection and enhancement effect at designated scale, orientation, location, and degree of enhancement. The images are then reconstructed by the inverse transform.

Figure 1 shown in section 1 illustrates the steerable pyramid transform on the chromosome image in Figure 4. Three levels of decomposition were performed. $\mathrm{A}, \mathrm{B}$, and $\mathrm{C}$ show the three bandpass filtered images at decomposition level 2, while D, E and $\mathrm{F}$ show them at decomposition level 3 respectively. The image $\mathrm{G}$ shows the downsampled lowpass image at decomposition level 3

Figure 4 contains two test images and their enhancement results. The upper left is the original chromosome image, and the lower left is the original image of a fabric with defects. The upper and lower right are the results of applying the selective detection and enhancement procedure to the corresponding original images. The chromosome image was enhanced to help visualize the dark and pale bands at different scales, while the fabric image was enhanced in order to detect and visualize the large horizontal and vertical defects in the texture. These objectives were clearly met as shown in the results. 


\section{:แI!}
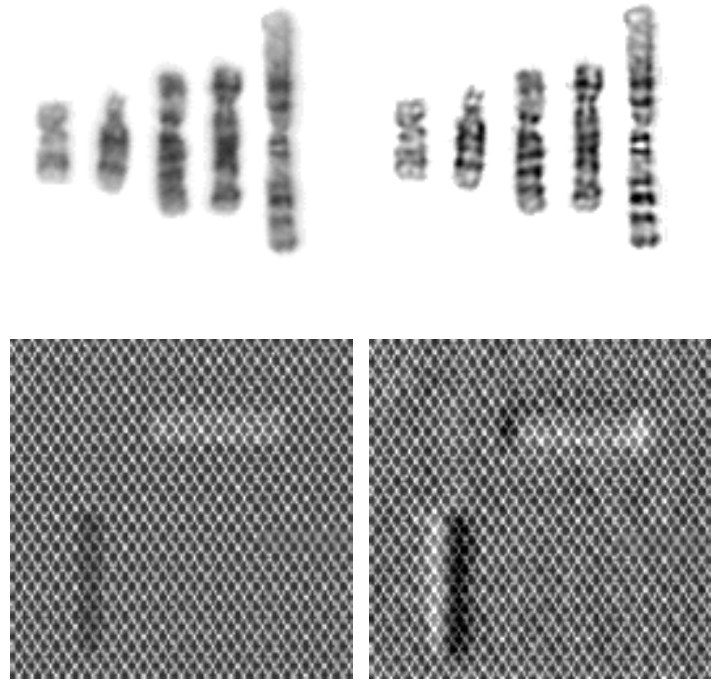

Figure 4. Experimental results of the proposed method. The original chromosome and fabric images are on the left. The images on the right are their enhancement results.

\section{Conclusions}

Selective detection and enhancement of image features are highly desirable for many image analysis applications. Traditional techniques based on conventional convolution filtering and contrast stretching are unable to facilitate this due to their intrinsic limitation. A multiscale image feature detection and enhancement method is proposed to overcome this limitation, and to achieve the desired capability. The proposed method utilizes a steerable pyramid transform, and exploits the image feature detection ability of the basis filters. Preliminary experimental results were favorable in establishing the feasibility of the method.

There are several advantages of this approach. First, it enables the user to interactively detect and enhance image features at selected scale, orientation, location, and degree of enhancement, thus offering flexibility for designated detail analysis. Second, image features can be located accurately and enhanced effectively because they are localized in both the space and transform domain representations, and the basis filters can be tailored to detection of the specific features. Third, unlike most multiscale image transforms, the representation is essentially aliasing-free and invariant to shift and rotation. The image feature detection and enhancement are consistent regardless of input image displacement. One disadvantage of the approach, however, is the substantial overcompleteness of the steerable pyramid transform $[3,4]$. The associated computational overhead could limit its suitability to real-time applications.

\section{Acknowledgments}

This research was supported in part by the National Institute of Child Health and Human Development under Grant No. 1R43HD33658-01.

\section{References}

[1]. W. T. Freeman and E. H. Adelson, "The Design and Use of Steerable Filters", IEEE Trans. PAMI-13(9):891-906, 1991.

[2]. E. P. Simoncelli, W. T. Freeman, E. H. Adelson, and D. J. Heeger, "Shiftable Multiscale Transforms", IEEE Trans. Information Theory 38(2):587-607, 1992.

[3]. E. P. Simoncelli, "Design of Multi-Dimensional Derivative Filters", in Proceedings of First International Conference on Image Processing, pp. 790-793, Austin, Texas, 1994.

[4]. E. P. Simoncelli and W. T. Freeman, "The Steerable Pyramid: A Flexible Architecture For Multiscale Derivative Computation", in Proceedings of Second International Conference on Image Processing, pp. 444-447, Washington D.C., 1995.

[5]. A. Karasaridis and E. P. Simoncelli, "A Filter Design Technique For Steerable Pyramid Image Transforms", in Proc. ICASSP-96, May, Atlanta, 1996.

[6]. K. R. Castleman, M. A. Schulze, and Q. Wu, "Simplified Design of Steerable Pyramid Filters", To appear in Proceedings of ISCAS'98, 1998. 